
internationales

vol. $21-n^{\circ} 3 \mid 2005$

Familles, destins personnels et appartenances collectives en migration

\title{
Enjeux de catégorisations et migrations internationales
}

Le Bureau International du Travail et les réfugiés (1925-1929)

\section{Dzovinar Kévonian}

\section{(2) OpenEdition \\ Journals}

\section{Édition électronique}

URL : https://journals.openedition.org/remi/2522

DOI : $10.4000 /$ remi.2522

ISSN : $1777-5418$

\section{Éditeur}

Université de Poitiers

\section{Édition imprimée}

Date de publication : 1 décembre 2005

Pagination : $95-124$

ISBN : 2-911627-45-8

ISSN : 0765-0752

\section{Référence électronique}

Dzovinar Kévonian, «Enjeux de catégorisations et migrations internationales 》, Revue européenne des migrations internationales [En ligne], vol. 21 - $n^{\circ} 3$ | 2005, mis en ligne le 01 décembre 2008, consulté le 15 avril 2022. URL : http://journals.openedition.org/remi/2522 ; DOI : https://doi.org/10.4000/remi. 2522

Ce document a été généré automatiquement le 15 avril 2022.

(c) Université de Poitiers 


\title{
Enjeux de catégorisations et migrations internationales
}

Le Bureau International du Travail et les réfugiés (1925-1929)

\author{
Dzovinar Kévonian
}

1 La question des enjeux de catégorisation a été ravivée en Europe depuis le début des années 1990 par une actualité croissante et souvent dramatique de la question de l'asile et des politiques migratoires. Les bouleversements à l'Est ont activé au sein de l'Europe communautaire un discours sécuritaire fondé sur la peur de flux massifs en provenance de la partie orientale du continent. Pour certains acteurs institutionnels le monde postbipolaire a été alors perçu comme celui du chaos, des dynamiques de fragmentation et d'un retour à un "âge de nature hobbesien" (Bigo, 1998). Dans le même temps, la construction de l'espace européen et les tentatives de poser les principes directeurs d'une politique d'asile commune se sont illustrés par des lieux de tension comme le centre de Sangatte et les difficultés liées à la non-concordance entre les espaces Schengen et Dublin. Le problème de la pérennité de la convention de 1951 s'est posé à travers la question des personnes déplacées, celle des flux mixtes, comme à travers la floraison de nouveaux statuts intermédiaires.

2 Ces éléments conjoncturels se sont conjugués au renouveau d'une histoire sociale s'interrogeant sur les constructions catégorielles et plus près de la présente thématique, des analyses menées par Gérard Noiriel. Ainsi, il nous a semblé profitable de proposer une réflexion sur la mise en perspective des enjeux de catégorisations concernant les réfugiés au niveau de l'action internationale à travers notamment le prisme des oscillations entre approche collective et individualisation. La démarche qui nous anime est celle d'une socio-histoire des relations internationales dépassant le cadre traditionnel du champ diplomatique, et permettant de saisir les enjeux d'une histoire politique et sociale de l'Europe au $\mathrm{xx}^{\mathrm{e}}$ siècle à travers l'étude d'un objet singulier et complexe, le réfugié. Celui-ci se présente à la charnière de plusieurs domaines: les relations internationales (statut du réfugié et apatride, diplomatie humanitaire, organisations internationales et non gouvernementales), l'histoire du droit (traitement de l'apatridie, expertise des juristes internationaux, déclarations de 
droits), l'histoire apolitique (droit d'asile, immigration), la sociologie culturelle (cadres mentaux et idéologiques normatifs). D'ailleurs, l'individualisation de l'objet singulier que constituent les réfugiés dans la première moitié du xxe siècle n'est pas le fait d'une construction théorique a posteriori. Elle constitue déjà, dans cette période, un enjeu politique et juridique au sein de la société internationale. Il s'agit donc moins de se pencher sur une donnée pré-construite que d'analyser la genèse, les processus d'individualisation, comme instruments et enjeux de «lutte des classements » (Pierre Bourdieu, 1998). Ces enjeux de catégorisations peuvent être appréhendés selon deux axes: le premier concerne l'évolution des catégories légales mises en place dans le cadre international depuis le premier conflit mondial, et le passage d'une approche collective (accèdent au statut de réfugiés des groupes nationaux définis) à un système qui individualise ce statut autour de la notion de persécution après 1951. Le second porte sur les enjeux de catégorisation verticale entre statut de réfugiés et ensemble plus vaste des statuts migratoires dont on le distingue. C'est cette dernière problématique qui sera ici développée à travers un exemple spécifique.

\section{Les enjeux de catégorisation verticale : réfugiés ou migrants?}

3 Les cadres généraux du problème posé par les réfugiés au début des années vingt sont affectés par une mutation quantitative et qualitative : apparition des « réfugiés de la paix ", ceux des traités de l'après-guerre en Europe et des échanges forcés de populations, massivité du phénomène, vide juridique existant du fait de l'extension considérable de l'apatridie et des dénationalisations forcées. La Société des Nations (ciaprès $\mathrm{SdN}$ ), nouvellement créée, en dépit $d u$ fait que les questions sociales n'appartiennent pas à ces priorités, devient néanmoins le lieu où s'élaborent des outils juridiques, où se développent des pratiques coopératives et ou les organisations privées et les milieux d'experts trouvent une tribune d'expression nouvelle dans un champ traditionnellement réservé aux acteurs étatiques. Dans le même temps, les processus socio-politiques, qui se jouent depuis le xIXe siècle en modifiant la triangulaire État/ Territoire/Population au profit d'une recomposition faisant de la logique d'appartenance ou de rejet un clivage dominant, changent les logiques de contrôle des mouvements migratoires et les critères qui définissent l'individu. La projection de ces conceptions sur le corps social amène la question des réfugiés à devenir le lieu de plusieurs tensions binaires : entre liberté de circulation et contrôle du territoire, entre national et étranger, entre droit de l'asile et politiques protectionnistes.

4 À partir de 1922, la SdN crée un certificat d'identité et de voyage pour les réfugiés russes, progressivement étendu à d'autres groupes nationaux. La définition du réfugié alors adoptée reconnaît l'existence de migrants forcés dépourvus de la protection juridique de leur État d'origine. L'assignation de la preuve du caractère forcé de la migration n'est pas à la charge du réfugié, l'appartenance au groupe national statutaire étant la condition nécessaire et suffisante d'accession à la catégorie. La désignation de la catégorie nationale accédant au statut de réfugié devient de fait le lieu central des enjeux dans cette période. Le certificat (communément connu sous le nom de "passeport Nansen ») accorde des droits limités au réfugié dans un cadre juridique rigide qui le distingue de la catégorie plus large du migrant économique. La définition, fondée sur une situation légale pénalisante - l'absence de nationalité rapportée à une 
causalité globalisante - la guerre mondiale, se conçoit en effet comme l'institutionnalisation d'une marginalité administrative et sociale. Or l'expérience menée par la SdN entre 1925 et 1929 consiste à transférer au Bureau International du Travail (ci-après BIT) l'action internationale menée en faveur des seuls réfugiés statutaires de cette période (russes et arméniens) et de fait à favoriser un chevauchement catégoriel entre réfugié et migrant. Comme on peut l'observer aujourd'hui (mais la remarque est également valable pour les années trente), ce chevauchement catégoriel a accentué en d'autres temps la fragilité structurelle du statut de l'exilé en introduisant l'argumentaire du vrai/faux réfugié. Par ailleurs, il ne présuppose pas pour cette période une meilleure considération du réfugié qui reste de tout temps stigmatisé et en butte à la suspicion du travestissement catégoriel. Quelle approche domine donc au sein du BIT ? En quoi peut-il mener une action en faveur des réfugiés statutaires? Ce chevauchement n'attesterait-il pas de la malléabilité de catégories juridiquement rigides et d'une variabilité de l'assignation identitaire en fonction de facteurs externes?

5 Au lendemain de la Première Guerre mondiale, les migrations économiques sont mises très tôt en connexion avec la question des réfugiés et la politique du droit de l'asile : de manière individuelle et privée par le patronat, de manière gouvernementale et intergouvernementale par le Bureau International du Travail et la pression des associations de contrôle des conditions migratoires. On retrouve là, en partie, l'héritage de la gestion migratoire intercontinentale de la période précédente, renouvelée par la demande de main-d'œuvre des pays touchés par les effets démographiques de la guerre mondiale. Le rôle prééminent de la France dans les années vingt dans l'adoption de ces mesures statutaires pour les réfugiés s'explique ainsi par le souci de favoriser un flux migratoire nécessaire à la reconstruction. Le transfert du Service des Réfugiés du HautCommissariat de la Société des Nations (dirigé depuis sa création en 1921 par Fridtjof Nansen) au Bureau International du Travail s'effectue en janvier 1925. Après une première phase dominée par l'urgence humanitaire et les tentatives de rapatriement des réfugiés ${ }^{1}$, le milieu des années vingt marque un tournant vers une politique de placements professionnels, par l'organisation d'une émigration secondaire des réfugiés russes et arméniens concentrés dans les pays limitrophes de leurs territoires d'origine. C'est donc à travers une analyse économico-sociale et non en termes d'asile que le BIT justifie la prise en charge de la question des réfugiés : "La guerre a eu comme conséquence le déracinement de grandes masses de populations contraintes de quitter leur pays natal ; arrachés à leur emploi habituel, les réfugiés sont devenus, sinon des sans-travail, du moins des éléments disqualifiés au point de vue économique. [...] Devant cette détresse, on commença par parer au plus pressé ; nourrir, vêtir et abriter les malheureux... [...] Toutefois, ce n'étaient là que des palliatifs temporaires. Tous ces déplacements d'après-guerre, coïncidant avec la crise économique d'après-guerre, ne pouvaient qu'aggraver la situation générale et si, entre-temps de nombreux réfugiés, plus ou moins assimilés, avaient réussi à se procurer du travail, beaucoup d'entre eux continuaient à dépendre de l'assistance publique ou privée $»^{2}$. Il est ensuite fait mention de l'inégale répartition des réfugiés et de la charge financière pesant de ce fait sur quelques États. Le principe de la solidarité internationale implique une meilleure répartition des réfugiés en Europe. Enfin, pour des raisons d'ordre sanitaire et social, la nécessité de transformer ces « centaines de milliers de chômeurs en producteurs utiles à la collectivité » s'impose. Dans les faits, lorsque Fridtjof Nansen soumet au Conseil de la SdN en juin 1924 sa proposition de transfert, il entend associer à l'œuvre des réfugiés 
une organisation à caractère permanent dotée de moyens structurels, ce dont lui ne dispose pas : ses mandats sont renouvelés annuellement et ses moyens sont dérisoires. Par ailleurs, en 1924, dans plusieurs pays, le problème posé par les réfugiés russes et arméniens s'est aggravé. C'est le cas de l'Allemagne, de la Yougoslavie, de la Bulgarie, de la Grèce, de la région d'Istanbul en Turquie et de la Chine (afflux des réfugiés russes). L'analyse de la situation en Allemagne au début de l'année 1925 par le délégué du BIT qui s'y trouve en poste fait également ressortir le caractère éminemment économique du problème des réfugiés. Isolant trois phases dans les flux de réfugiés, il distingue : jusqu'en 1923, la grande vague d'émigration politique, l'Allemagne étant devenue pour des raisons géographiques, politiques et surtout économiques le lieu de plus forte concentration en Europe occidentale; à partir de la fin de l'année 1923, le grand mouvement de départ en particulier vers la France en raison de la crise économique et financière ; à partir de 1925, la stabilisation de la présence des réfugiés dans le pays. Il explique : «Lorsqu'on étudie le caractère de ces mouvements, on ne peut échapper à la conclusion que leur facteur principal est de nature purement économique, à tel point que la réglementation gouvernementale se trouva jusqu'à présent impuissante à contrôler efficacement les déplacements des réfugiés $»^{3}$. Le ralentissement des migrations secondaires des réfugiés est donc à chercher dans leur stabilisation professionnelle. Enfin, la dernière argumentation repose sur l'idée du danger politique et social que peuvent représenter des réfugiés sans travail. L'intégration économique est conçue comme agent actif d'intégration sociale.

Le mandat accepté par le BIT à l'automne 1924, et qui prend effet à partir de janvier 1925 , comprend donc la recherche, la coordination et la communication des offres de placement qui peuvent être offertes aux réfugiés, des conditions dans lesquelles les réfugiés peuvent profiter de ces offres, une appréciation du nombre de réfugiés à placer. Comme l'écrit quelques mois plus tard, Albert Thomas, le célèbre directeur du BIT, le Bureau «accomplit un travail de caractère purement technique, qui fait de lui une chambre de compensation internationale pour les offres et les demandes d'emploi des réfugiés dans les divers pays $»^{4}$. Or, il est évident que les réfugiés ne sont pas des migrants comme les autres et les problèmes posés par leur condition sont spécifiques et nombreux: masse élevée et non identifiée de populations concernées; problème de répartition entre les pays de concentration et les pays de travail disponible; question de leur statut d'apatride et de la nécessité de les doter de titres d'identité et de voyage ; obtention de visas pour des personnes sans nationalité donc sans possibilité de retour dans leur État d'origine; nécessité d'assurer leur placement préalable dans les pays d'accueil avant d'organiser leur transfert; problème de leur incapacité financière de couvrir leurs frais de transport.

7 Dès novembre 1924, Albert Thomas réunit les délégués nationaux du BIT à Genève et organise le travail de collecte des données statistiques concernant les réfugiés. Il propose également d'organiser sur le modèle du Comité consultatif des organisations privées près le Haut-Commissaire Nansen à Genève, la collaboration des comités consultatifs locaux avec le représentant du BIT. Au niveau structurel, le BIT dispose donc du petit Service des réfugiés mis en place par Nansen et dirigé par le major T. F. Johnson. Ce service travaille en étroite collaboration avec le Service des Migrations, dirigé par Louis Varlez. Plus importante est la mise à disposition pour ce programme des bureaux et correspondants nationaux du BIT dans les différents pays : Allemagne, Autriche, Belgique, Bulgarie, Chine, Espagne, États-Unis, Finlande, France, Grande- 
Bretagne, Grèce, Hongrie, Italie, Japon, Pologne, Pays Baltes, Syrie-Liban, Tchécoslovaquie, Turquie, Yougoslavie.

\section{Classer, identifier, quantifier les réfugiés « sans emploi »}

8 Le premier objet du recensement du BIT est de fixer définitivement le nombre des réfugiés sans emploi tout en étant capables de gagner leur vie «par leurs propres efforts ». Pour se faire, le Service des réfugiés met au point un questionnaire-type qui est adressé à tous les délégués afin qu'ils le soumettent individuellement aux réfugiés. Dans ce document, la France est le seul État européen à être représenté sur la liste de destinations possibles. Pour le reste, le continent américain est le plus largement présent. L'ensemble des réponses communiquées par les différents délégués est présenté devant l'Assemblée de la SdN en septembre 1925 : sur environ un million de réfugiés russes et arméniens comptabilisés, 256633 sont «sans emploi ». Des données complémentaires obtenues quelques mois plus tard portent ce total à 298594 réfugiés statutaires.

9 Une catégorisation précise par profession est adoptée pour l'ensemble des pays considérés, selon les critères déjà en vigueur au BIT. Les résultats donnent donc un tableau synoptique impressionnant par la maitrise apparente de l'identification : 25 catégories et 14 sous-catégories sont représentées. Pourtant, les catégories génériques non qualifiées regroupent l'essentiel des réfugiés comptabilisés, ce qui rend compte des limites de la démarche statistique adoptée et du non-ajustement des catégories proposées aux parcours et profils individuels. Quelle portée accorder à ces données et à cette volonté de maîtrise statistique de cette population flottante de réfugiés? L'objectif est pragmatique et implique un traitement individuel d'une situation collective: mettre en vis-à-vis des demandes d'emplois précises et des migrantsréfugiés au profil correspondant. En vérité, l'enquête préalable a reposé dans chaque délégation du BIT sur une déclaration volontaire des réfugiés qui ont alors rempli la fiche individuelle et ont été de ce fait comptabilisés comme sans emploi et favorables à un transfert. Ces éléments limitent de fait la validité des résultats sur laquelle repose l'opération de quantification et de classement.

10 Prenons l'exemple du travail mené par Henry Reymond puis par le Dr Heimroth en 1925-1926 comme chefs de la délégation de Vienne. La caractéristique de cet État dans ces années est de constituer un pays essentiellement de transit pour les réfugiés. Vienne est la porte qui conduit des Balkans en Europe occidentale. Le problème, explique H. Reymond est l'émigration illégale et individuelle : les illégaux ne peuvent être expulsés (puisque les États voisins ne les accueillent pas sans document en règle ni visa) et ne peuvent non plus être «enfermés » car le coût est trop élevé pour l'État autrichien. Beaucoup de réfugiés russes arrivent en Autriche de différents pays sans visa autrichien et ils quittent le pays sans visa du pays de destination. C'est, explique-til, la suite naturelle des difficultés faites de la part des différents États à la migration légale des réfugiés, d'où une augmentation de la migration clandestine : «Ils voyagent souvent à pieds ou traversent au moins à pieds les frontières $»^{5}$. H. Reymond souligne d'ailleurs qu'il n'est absolument pas en mesure de soumettre une statistique de ce flux migratoire. D'autre part, résident en Autriche un grand nombre de réfugiés qui malgré toutes les lois et règlements concernant les passeports, ne sont pas connus de la Délégation et ne disposent donc ni du certificat d'identité et de voyage (passeport 
Nansen), ni d'aucun passeport valable. Ces réfugiés possèdent tout au plus un ancien passeport impérial russe, mais le plus souvent, ils n'ont qu'un certificat de baptême russe ou la connaissance de la langue russe est leur unique légitimation. Ce manque de documents ne pose pas de problème localement car la police locale les connaît et ils possèdent leur « Meldezettel » (liste des étrangers). Par ailleurs, l'obtention du passeport Nansen est conditionnée par la perte de la nationalité russe (statut de réfugié-apatride). Or, une partie des réfugiés répugnent à se procurer l'attestation de la Légation soviétique selon laquelle ils ne sont pas ressortissants de l'Union soviétique, document nécessaire pour devenir réfugié statutaire: "On ne veut pas se barrer le chemin du retour en Russie... On a besoin du passeport Nansen, simplement pour pouvoir voyager en Europe, mais on ne peut pas savoir comment les affaires tourneront, on préfère donc ne pas se priver du retour chez les Soviets $»^{6}$. Une autre source parle également des hésitations des réfugiés à venir s'inscrire dans les délégations en raison de la crainte que cette formalité soit le prélude à des mesures d'expulsion ou de répression. L'opération de recensement demandé par le BIT a donc présenté de grandes difficultés de réalisation, sans compter les incompréhensions entre les demandes formulées par Genève et les données présentées par la Délégation viennoise, qui ne conviennent pas au Service des Réfugiés. L'année 1925 est émaillée de courriers de rappel et de demandes de rectification des données statistiques que $\mathrm{H}$. Reymond avoue ne pas comprendre. On peut ainsi observer les limites de cette triple catégorisation : catégorie du statut personnel de réfugiés russes, catégorie de sans-emploi et catégorie professionnelle.

11 La délégation allemande rencontre des difficultés plus grandes encore dans sa tentative d'établir des statistiques concernant les réfugiés russes qui ont afflué en masse au début des années vingt. Un total de 800000 réfugiés russes est proposé pour l'année 1923, "mais il est permis de douter qu'il ait été atteint", ajoute le délégué, A. Scherbatskoy. Quant aux chiffres cités au Reichstag par les représentants élus, les écarts sont grands et les variations nombreuses, selon la «thèse » soutenue par tel ou tel parti. Pour l'année 1925, l'évaluation établie par le ministère allemand de l'Intérieur fait état d'environ 400000 réfugiés russes ${ }^{7}$. Quant au nombre de réfugiés sans emploi, aucun moyen de les comptabiliser n'existe vraiment. Les autorités régionales ne quantifient que ceux des chômeurs qui sont à la charge de l'assistance publique. Or les réfugiés russes chômeurs ne bénéficient pas d'allocation régulière. Le délégué estime donc de son propre chef et après consultation des organisations privées, le nombre de réfugiés sans emploi à 80000 en hiver, surtout des agriculteurs saisonniers, qui à la différence des Polonais ne peuvent repartir pendant la saison hivernale. Lorsqu'en 1928 , le Service des réfugiés réitère ses demandes dans le contexte difficile de la pérennité de l'œuvre internationale en faveur des réfugiés, $M$. Schlesinger explique que son impossibilité à fournir des données s'explique par l'absence de centralisation et d'une structure commune aux différents Länder. Ce n'est en effet qu'en 1934 que le pays connaît la mise en place d'une structure centralisée, l'office statistique unifié de l'État nazi.

12 Cette logique statistique, au sein du BIT, est assez communément employée dans le domaine des migrations et surtout du chômage. On retrouve là l'héritage de la statistique française et notamment de la Statistique Générale de France, dirigée par Lucien March, qui a acquis sa légitimité par des interventions sur le domaine du travail et de la main-d'œuvre à partir des années 1890 . On sait par ailleurs, que ce milieu des statisticiens français s'est lié à un réseau de fonctionnaires, intellectuels, responsables 
syndicaux et politiques parmi lesquels on trouve Alexandre Millerand, François Simiand, Arthur Fontaine, Albert Thomas, etc. D'autre part, lorsqu'en 1891 est créé l'Office du Travail au sein du ministère du Commerce, Arthur Fontaine en a pris la tête et la SGF lui a alors été rattachée. Deux décennies plus tard, les liens existants entre statisticiens et responsables politiques ont été renforcés pendant la guerre au sein du cabinet Albert Thomas au ministère de l'Armement. Le début des années vingt a vu en France ces liens se distendre et l'influence des statisticiens se réduire. Parmi les raisons avancées par Alain Desrosières pour expliquer ce repli, on trouve des causes propres à la SGF mais également le poids d'une conception libérale de l'État qui domine jusqu'aux années trente, les expériences menées pendant la guerre par Clémentel et Thomas n'ayant constitué que des exceptions circonstancielles. Or, il n'est pas sans effet de constater que le BIT est dans les années vingt le lieu où ce réseau s'est en partie reconstitué. Arthur Fontaine préside le conseil d'administration du BIT, Albert Thomas en est le directeur. D'ailleurs, les travaux d'harmonisation des nomenclatures et des méthodes entrepris au sein de l'Institut International de Statistique depuis 1895, sont poursuivis au lendemain de la guerre par le BIT. La collection Études et Documents publiée par celui-ci en atteste largement dans ses séries $\mathrm{N}$ (statistiques), $\mathrm{O}$ (migrations) ou C (chômage). La démarche entreprise en matière de réfugiés constitue donc une extension d'une approche existante, par un croisement entre statistique des mouvements migratoires et statistique du travail.

\section{La « normalisation » du statut de réfugié, condition du placement professionnel}

13 Dans cette perspective économique, le réfugié apatride ne représente pas une catégorie à part. Or il l'est pourtant juridiquement en ne disposant pas des mêmes droits que les autres migrants. Le "passeport Nansen» délivré aux réfugiés russes et arméniens (seules catégories nationales alors reconnues par la $\mathrm{SdN}$ ) permet d'attester de leur identité et de leur statut personnel, et en principe de pouvoir voyager. En 1925, la reconnaissance de ce document est assez large puisqu'elle concerne 40 États pour les réfugiés russes et 28 pour les réfugiés arméniens. Mais, en vérité, la plupart des pays ne le mettent pas en application et dans la pratique, les réfugiés n'accèdent pas au statut. Parmi les raisons qui expliquent le peu d'application réelle du certificat, domine la méfiance des autorités à l'égard des populations réfugiées et l'impossibilité où elles disent se trouver de « distinguer entre les demandes de réfugiés de bonne foi et celles de personnes qui, quoique ayant droit à un certificat, avaient réussi à obtenir la délivrance d'un certificat pour des buts irréguliers $»^{8}$. On retrouve là une argumentation familière de stigmatisation du réfugié qui même "vrai » est assimilé à un danger politique et social. Ainsi, en 1926, quatorze gouvernements disent appliquer le statut international et le nombre de certificats délivrés s'élèvent à 155000 seulement. Or, ce certificat d'identité est indispensable comme document de voyage pour tout réfugié sans travail auquel le BIT va procurer un emploi selon le nouveau système de placement qui se met en place. Par ailleurs, la délivrance du certificat, les visas d'entrée et de transit sont payants et les tarifs varient grandement d'un État à l'autre (entre 0 et 52 francs suisses selon les données du Service des Réfugiés). Enfin, le réfugié, selon l'arrangement de 1922 qui fixe les conditions de délivrance du certificat d'identité est soumis à des contraintes dans sa liberté de circuler d'un pays à l'autre, en 
particulier la non-reconnaissance du droit au retour dans le pays qui a délivré le certificat.

14 Ces conditions s'expliquent par la perspective dans laquelle à l'origine le certificat a été élaboré et qui envisageait la mise en place de ce document d'identité et de voyage dans le but de désengorger les zones de concentration de réfugiés, situées à proximité des pays d'origine en favorisant une émigration secondaire. L'idée n'est donc pas d'attribuer aux réfugiés un document leur permettant de circuler en dépit de leur absence de nationalité. D'ailleurs l'expression même de passeport est récusée par tous les États, qui n'entendent pas favoriser une confusion des genres nuisibles au contrôle de la circulation migratoire. Par ailleurs, l'idée de ne pas accorder au réfugié le droit au retour dans l'État qui lui a accordé le certificat vise à établir qu'aucun engagement ni responsabilité ne lient l'État qui a établi le document et son porteur. En particulier, en cas d'expulsion, il n'existe aucune obligation d'accueillir le réfugié. Cet état de fait représente un obstacle pour la politique de placement du BIT. Ainsi, c'est par des accords bilatéraux conclus avec les gouvernements allemands et grecs qui ont accepté le principe du rapatriement des réfugiés indésirables que le BIT a réussi à négocier en 1925 avec le gouvernement canadien une opération de placement professionnel. Lucien Wolf, célèbre représentant du Joint Foreign Committee de Londres, membre actif du Comité consultatif des organisations privées du Haut-Commissariat pour les réfugiés, donne plusieurs exemples concrets d'opérations d'émigration qui ont échoué en raison de ce statut précaire du certificat d'identité et de voyage pour réfugiés-apatrides. Le dernier problème posé au BIT est celui des moyens financiers nécessaires aux opérations de transport des réfugiés vers les pays où des contrats de placement ont été trouvés. Les réfugiés n'ont, dans leur grande majorité, pas les ressources leur permettant de payer les compagnies de transport et de navigation (dans la perspective des placements outre-mer), ni les frais de visas de transit et d'entrée. Où trouver les ressources correspondantes, alors même que la SdN n'alloue annuellement au Service des réfugiés qu'une somme modique, à peine suffisante pour supporter les frais de fonctionnement? Obstacles administratifs, juridiques et financiers se conjuguent, montrant la distance réelle séparant le réfugié-apatride du migrant ordinaire. Le HautCommissaire pour les réfugiés, Fridtjof Nansen et Albert Thomas vont donc s'attacher à «normaliser » le statut de réfugié en proposant, à l'automne 1925, la réunion d'une conférence intergouvernementale où seront étudiées les possibilités d'amélioration des certificats d'identité des réfugiés et la création d'un fonds de roulement de $100000 \mathrm{E}$ qui faciliterait leur placement.

15 L'Arrangement relatif à la délivrance de certificats d'identité aux réfugiés russes et arméniens conclu en mai 1926 à l'issue de la conférence intergouvernementale contient une première résolution par laquelle la définition de la qualité de réfugié pour les Russes et Arméniens est précisée ${ }^{9}$. La catégorie des réfugiés statutaires est définie par rapport à l'État d'origine et par rapport à une nationalité ou communauté nationale. Ces deux critères ignorent la causalité politique de l'exil et écartent de la définition les apatrides de fait ${ }^{10}$. L'Arrangement de 1926 comprend également plusieurs points importants consacrés aux moyens de faciliter « la liberté de déplacement » des réfugiés. Est adopté le principe de l'apposition sur les certificats d'identité d'un visa de retour pour les réfugiés statutaires quittant le pays qui leur a accordé le certificat. Les enfants de moins de quinze ans sont admis à être inscrits sur le certificat de leurs parents. Ceci reflète une nouvelle réalité de l'exil qui concerne la constitution ou reconstitution des 
familles et la nature d'une mobilité migratoire familiale. À la demande du BIT, la Conférence recommande que les réfugiés indigents bénéficient de la gratuité des visas et que les sollicitations du Bureau en matière de facilités de transports des réfugiés soient considérées favorablement. Enfin, l'Arrangement de 1926 institue une taxe spéciale de cinq Francs-or au profit d'un fonds de roulement institué par la SdN et destiné à financer les programmes de placements professionnels des réfugiés ${ }^{11}$. En 1926, l'Arrangement entre officiellement en vigueur en Autriche, en Belgique, en Bulgarie, à Cuba, au Danemark, en Estonie, en France, en Grèce, en Hongrie, en Inde, en Pologne et en Suisse. En 1927, l'Allemagne, la Finlande, l'Irlande, le Luxembourg, la Norvège et la Suède le ratifient également. La Roumanie et la Yougoslavie y adhèrent l'année suivante $^{12}$. La Grande-Bretagne, pourtant signataire, ne le ratifie pas. La nonimplication politique de la Grande-Bretagne en ce qui concerne la question des réfugiés est une constante durant toutes les années vingt, en particulier sous les cabinets conservateurs entre 1924 et 1929.

Les résultats de la conférence semblent donc aller dans le sens voulu par F. Nansen et A. Thomas. Néanmoins, les comptes rendus des débats montrent les limites de l'accord. La majorité des États ont refusé d'appliquer le principe d'une attribution obligatoire du certificat pour les réfugiés russes et arméniens et donc d'une uniformisation de leur statut. Ceux-ci peuvent en faire la demande s'ils le souhaitent, mais dans la plupart des pays, ils sont porteurs soit d'un certificat d'identité spécial (le «Personalausweis » en Allemagne, le " passeport pour étranger » en Suisse, etc.), soit d'un permis de séjour et d'une carte d'identité. La tendance majoritaire, dont la France se fait le porte-parole, est de ne considérer le certificat que comme un titre de voyage pour les réfugiés et non comme un papier d'identité. Ce contre quoi Fridtjof Nansen s'insurge vainement en rappelant la teneur de l'arrangement de 1922. Le second point qui est rejeté par la conférence est celui d'une uniformisation ou mieux d'une suppression des taxes nationales prélevées sur le certificat lors de son attribution. Enfin, la grande majorité des délégations tombent d'accord pour récuser la proposition soumise de constituer de commissions nationales comprenant des représentants qualifiés des réfugiés pour assister les autorités dans les procédures les concernant. Enfin, la conférence laisse apparaître la distance qui sépare les pays européens des pays d'outre-mer dans la question des modalités du contrôle des migrations. Le représentant du Canada estime en l'espèce que dans toutes ces discussions, il n'est pas assez tenu compte du point de vue des pays d'immigration d'outre-mer. Ainsi, au Canada, il n'existe pas de système de surveillance des étrangers comparable à celui appliqué dans la plupart des pays européens au moyen de permis de séjour. L'idée de prélever une taxe annuellement pour alimenter le fonds de roulement est très difficile en raison même de l'étendue du territoire et de la liberté de mouvement laissée aux étrangers qui ont pénétré dans le pays. On retrouve là la référence aux distorsions existantes entre les différents systèmes de limitation de la circulation migratoire.

Les limites énumérées ci-dessus relèvent d'une distinction structurelle bien identifiée entre domaine réservé de la souveraineté étatique et espace contractuel international. Nulle surprise donc à voir la conférence récuser toutes les propositions pouvant affecter cette sphère réservée qui englobe le contrôle de la circulation migratoire, l'identification et la gestion administrative des étrangers. Les stipulations de l'Arrangement de 1926 vont-ils permettre au BIT de mettre en place sa politique de placement? La réponse sera mesurée. L'application des résolutions sera aussi variable d'un pays à l'autre. Néanmoins, sur la base de cet accord, le BIT va conclure des 
arrangements ponctuels avec certains États concernés par les réfugiés et leurs transferts améliorant leur condition ou le coût de leur placement dont l'exemption de mesures restrictives visant les étrangers ${ }^{13}$, l'octroi de réduction sur les tarifs des transports, l'extension du système du certificat $\mathrm{Nansen}^{14}$, la gratuité ou réduction des droits sur les visas de transit ou d'entrée. Le BIT obtient également des subsides gouvernementaux pour certaines délégations. Enfin, en Autriche, Bulgarie et Grèce, des arrangements interviennent pour que la délivrance de certificats et d'actes d'état civil, se fasse seulement sur recommandation du délégué après examen de chaque demande en collaboration avec la commission consultative locale de réfugiés.

\section{Une « chambre de compensation internationale » entre l'offre et la demande d'emploi des réfugiés}

18 La perspective dans laquelle se place le BIT implique non seulement de quantifier les réfugiés sans emplois mais également de prendre connaissance de l'offre existante. Avant la nouvelle organisation de 1925, le BIT avait déjà été sollicité à plusieurs reprises pour informer le Service des réfugiés des possibilités de placement. Les opérations conclues concernaient alors essentiellement la France. L'idée qui prévaut en 1925 est qu'étant donné la situation économique difficile et la « crise du chômage » en Europe, les débouchés sont à chercher, en dehors de l'exception française, dans les pays d'outre-mer, en Amérique du Sud. Albert Thomas se rend sur place durant l'été 1925 et une mission composée d'experts parmi lesquels figure Louis Varlez (le chef du Service des migrations du BIT) va passer cinq mois en Argentine, au Brésil, au Paraguay et en Uruguay. Des représentants de la Jewish Colonisation Association et de la Conférence Universelle Juive sont également associés à la mission à titre consultatif. Leur présence s'explique par les contacts étroits noués entre le Service des Réfugiés et Lucien Wolf, et par l'expérience acquise par la Jewish Colonisation Association en Argentine. Selon le chef de mission, à travers une argumentation macro-sociale assez rudimentaire, le chômage est l'une des causes principales d'agitation politique. Il est aggravé voire dominé, dans certains pays, par le problème des réfugiés. La solution réside donc dans la mise en valeur des régions les moins peuplées du monde et la migration de l'excédent des populations des régions surpeuplées. De plus l'État d'émigration profiterait ainsi de l'expansion de ses marchés d'outre-mer ${ }^{15}$.

19 Le rapport de mission conclut à l'existence d'une demande importante de maind'œuvre à la fois agricole et industrielle et souligne les bonnes dispositions des représentants étatiques rencontrés, en particuliers brésiliens (propositions précises de placements des États de Rio de Janeiro et de Sao Paulo). Si le principe lui-même est donc accueilli favorablement, une exigence impérative est posée par les interlocuteurs sud-américains : la sélection et le contrôle en amont des réfugiés. Le réfugié, répondant aux critères économiques, doit se soumettre à ce «droit de conservation de l'État » qui le conduit à sélectionner les immigrants selon certains critères que Paul Fauchille énonce dans son étude de 1924 : «Les étrangers à écarter seront donc spécialement ceux qui sont atteints de maladies contagieuses ou héréditaires ; [...] ceux qui, par leur immoralité ou leurs antécédents judiciaires peuvent être une cause de trouble de l'ordre public ; ceux qui peuvent introduire dans les pays des doctrines subversives et anarchistes». La mise en place des opérations de placement nécessite un bon fonctionnement des délégations du BIT en Europe et l'établissement de représentants 
permanents, l'un à Buenos Aires et l'autre à Rio de Janeiro. L'année suivante, ces représentations sont créées et prospectent en vue de présenter aux États intéressés des offres concrètes. En 1927, le BIT organise une réunion intergouvernementale à Genève, consacrée spécifiquement à la question du placement dans les pays d'Amérique du $\mathrm{Sud}^{16}$. Des offres concernant près de 30000 réfugiés russes sont présentées ainsi que des études de contrats de colonisation en Argentine, Bolivie, Brésil, Paraguay, Pérou et à Saint-Domingue. La position de la France, lors de cette réunion, est d'être en retrait par rapport au programme de placement outre-mer, ne souhaitant pas voir partir les réfugiés alors présents sur son territoire et ne souhaitant pas non plus voir se tarir une source sélectionnée de main-d'œuvre dont elle était jusque-là l'essentielle bénéficiaire.

Le projet de placement d'un nombre important de réfugiés sur le continent sudaméricain va occasionner une dépense d'énergie considérable durant les années 1926-1927. Néanmoins, le bilan est décevant, comme l'explique Albert Thomas en 1928. En matière de formalités d'entrées sur le territoire argentin, les réfugiés ont pâti de la difficulté à présenter un certificat de bonne conduite attestant que le titulaire n'a pas durant les cinq années précédentes fait l'objet de poursuites judiciaires. En outre l'État a refusé de renouveler le passeport Nansen. Les difficultés financières ont constitué une autre cause de l'échec du projet sud-américain : les agents du BIT ne parviennent pas à obtenir de contrats de travail auprès des employeurs. D'autres raisons viennent s'ajouter : l'opposition des autorités locales à favoriser le placement de réfugiés comme immigrants et le renforcement du contrôle de l'immigration à partir de 1925 au Brésil comme en Argentine. Le bilan global des opérations de placements mises en place par le BIT pour les réfugiés pour les deux premières années donne des résultats qu'Albert Thomas présente néanmoins comme concluants : 35300 réfugiés ont été placés en 1925 et 1926, mais en vérité, pour $52 \%$ d'entre eux, ils l'ont été en France ${ }^{17}$.

21 Le placement de 35000 réfugiés a coûté «environ 600000 francs »: les réfugiés pourvoyant au transfert grâce au fonds de roulement. Par ailleurs, la référence à la variable du chômage en Europe permet un autre calcul avantageux: le coût d'un chômeur en 1926 s'élève à 500 Francs-or par tête, alors que le placement des réfugiés revient à 17 Francs-or par tête. Enfin, le rapport intègre aussi d'autres avantages : « Si l'on considère les frais d'administration comme une sorte de prime internationale d'assurance contre les troubles politiques et sociaux inhérents à tous les problèmes de cette catégorie, la transformation de réfugiés indigents dont l'entretien incombait aux fonds publics en facteurs économiques positifs pour une somme aussi minime paraîtra encore plus significative ${ }^{18}$. Cette tendance matérialisée par le BIT en faveur d'une assimilation du réfugié au migrant économique coexiste avec une catégorisation subjective qui fonde une perception négative des réfugiés, groupements ou individus considérés comme dangereux. L'étude de la juriste Madeleine de Bryas, parue en 1926 sous le titre: Les peuples en marche: Les migrations politiques et économiques en Europe depuis la guerre mondiale permet d'illustrer ce point de vue largement répandu : «Alors que l'émigrant ordinaire transporte avec lui son capital, une grande partie de ces réfugiés ont fui hors de chez eux sans rien emporter [...]. Ils constituent les débris humains de la catastrophe européenne, qui ont été recueillis par d'autres pays que le leur. Ce travail d'absorption est infiniment pénible et coûteux, et a aggravé certainement l'état économique du continent. La surabondance de la population étrangère dans certains centres a causé des troubles, en accentuant la crise du chômage 
et du logement, et en augmentant le nombre d'indigents qui vivent de la bienfaisance privée ou publique $»^{19}$.

La valeur économique d'un "débris humain» est évidemment très relative. Il faut l'interpréter comme l'utilisation d'un argumentaire destiné à faire écho aux discours sécuritaires étatiques pour justifier le renouvellement d'un budget qui fait tous les ans à l'Assemblée de la SdN l'objet d'oppositions et désarmer au sein même du BIT ceux qui n'étaient pas favorables au transfert du Service des réfugiés. En 1928, Albert Thomas considère que les résultats des opérations de placement pour les années 1927 et 1928 sont médiocres. Ils s'expliquent à la fois par la crise que connaît la France en 1927, et par l'échec des projets sud-américains. Seulement 14600 réfugiés ont été placés durant ces deux années. Au total 50000 personnes ont bénéficié entre 1925 et 1928 des opérations de placement. Au terme du mandat du BIT on peut remarquer que les données rassemblées par le Service des Réfugiés n'ont pas gagné en exactitude au fil des ans. En outre aucune donnée n'est disponible pour l'Allemagne, la Grande-Bretagne et la Belgique en 1928. Des évaluations sommaires et non vérifiables sont présentées pour la France. Mais ceci n'empêche pas le directeur du BIT de considérer pour la période considérée que le nombre de réfugiés statutaires chômeurs serait passé de 400000 (évaluation en distorsion avec les données présentées en 1925) à 200000 personnes ${ }^{20}$.

23 Parmi les difficultés rencontrées, outre les problèmes spécifiques concernant les projets de colonisation en Amérique du Sud, le Service des réfugiés a été confronté aux mesures d'expulsion de réfugiés, adoptées par le gouvernement turc au milieu des années vingt. Ces décisions qui visaient près de 2500 réfugiés russes ont provoqué la mobilisation du BIT et l'ont conduit à s'engager à placer les réfugiés expulsés dans d'autres pays. En acceptant un tel programme d'urgence, Albert Thomas illustre les ambiguïtés du traitement philanthropique et social d'une question qui se pose en réalité en termes de droit d'asile et du respect de la personne humaine. Il est d'ailleurs intéressant de noter qu'après des efforts, le BIT finit par trouver des solutions de placements pour ces réfugiés expulsés mais il se heurte au refus de ces derniers d'accepter les contrats de travail proposés ${ }^{21}$. Enfin, le rapport de 1928 explique que les compétences du BIT ont été utilisées avec succès tant que des ouvriers et des agriculteurs pouvaient être placés. Or, parmi les 200000 réfugiés chômeurs restants, figurent de nombreux membres de professions libérales, pour lesquels les débouchés sont en Europe (et en France notamment) quasiment inexistants. Figurent également des invalides, des vieillards et des enfants pour lesquels, là encore, le BIT ne peut agir. C'est donc, au terme de ce constat que le directeur du BIT propose la fin de la prise en charge de la question du placement par l'institution qu'il dirige.

Enfin, il est intéressant d'évaluer la réception auprès des réfugiés concernés de la politique de placement adoptée en leur faveur au titre de migrants et les effets en retour de ce chevauchement catégoriel. Ainsi, les placements en Amérique du Sud n'ont pas rencontré en Bulgarie et en Yougoslavie un grand engouement. Raymond Schlemmer, délégué du Service des Réfugiés pour le Proche-Orient, aborde la question dans sa correspondance avec Genève. Très peu de réfugiés russes, actuellement dans les Balkans, sont disposés à aller en Amérique du Sud. En août 1927 il écrit : «Tous ont l'espérance de rentrer un jour en Russie et répugnent à l'idée de mettre l'océan entre eux et leur patrie et de s'attacher par des liens de propriétés, d'obligations hypothécaires, de contrats à longue échéance à un pays d'exil » ${ }^{22}$. Schlemmer se 
demande d'ailleurs si pour les Balkans, il faut vraiment insister pour un placement outre-mer au risque d'un "fiasco faute d'émigrants». Le pays de destination majoritairement envisagé est la France explique-t-il et pour l'Amérique du Sud, la seule solution est de procéder à un recensement et d'embarquer immédiatement les réfugiés qui s'inscriront. Par ailleurs les réfugiés rechignent à payer le prix du timbre apposé sur le passeport Nansen, estimant qu'ils ne savent pas où va leur argent. Enfin, en ce qui concerne les réfugiés russes, le Haut-Commissaire pour les réfugiés Fridtjof Nansen est accusé de sympathies pour le régime soviétique. Ainsi pour nombre d'organisations de l'émigration russe comme pour de nombreux réfugiés, adopter le passeport Nansen, c'est accepter la situation d'apatridie, dépolitiser un exil conçu encore comme provisoire, faisant le jeu d'un régime qui a fait de la dénationalisation pour motif politique un moyen d'expulsion commode. D'ailleurs, Schlemmer considère qu'il faudrait mettre un autre symbole sur le passeport, une mappemonde ou une figure allégorique: "Je crois qu'on rectifierait une erreur psychologique qui faciliterait grandement l'acquisition sans résistance des dits timbres Nansen ${ }^{23}$.

\section{Un système qui fonctionne au profit de la France}

La majorité des réfugiés placés par l'intermédiaire du BIT avant 1925 l'ont été en France. Les placements avaient fonctionné à partir d'offres précises émanant des services publics ou de sociétés privées d'immigration. Lorsqu'en 1925, Albert Thomas met en place son système international de placement des réfugiés, il entend généraliser le modèle « français » d'immigration dirigée. Ainsi, l'exemple donné à l'Assemblée de la SdN de 1926 dans le rapport annuel est celui du transfert d'un convoi de réfugiés russes résidant en Yougoslavie, appelés à travailler dans une usine chimique en France: communication de la proposition d'emploi par le Service de la main-d'œuvre étrangère du ministère français du Travail ; questionnaires individuels remplis par les réfugiés à Belgrade; réunion d'une commission de sélection composée du délégué local, de représentants des organisations de réfugiés, de conseillers agricoles et commerciaux; visite médicale obligatoire pour les réfugiés sélectionnés; enquêtes de moralité; distribution des certificats Nansen. Le rapport conclut en ces termes: "Le convoi parcourut une distance totale de $1758 \mathrm{~km}$. Grâce aux facilités indiquées ci-dessus, le montant total des frais pour chacun des réfugiés n'a pas dépassé 150 francs français ».

Quelle est la nature de l'accord conclu en 1925 avec la France? Il s'agit en fait de deux accords l'un avec M. Paon, chef du Service de la main-d'œuvre agricole au ministère de l'Agriculture et l'autre avec $M$. Lebelle, chef du service de la main-d'œuvre étrangère du ministère du Travail. Après une rencontre entre M. Lebelle et $\mathrm{A}$. Thomas en janvier 1925, il est décidé que les réfugiés russes seront recrutés par l'entremise du Service des Réfugiés du BIT. Quelques semaines plus tard, c'est Jean Duhamel de la Société Générale d'Immigration (ci-après SGI) qui est reçu par Albert Thomas. Un arrangement complémentaire est trouvé : la SGI recrutera sur place les réfugiés en collaboration avec les délégués locaux du BIT avec l'accord de M. Lebelle. "Peut-être peu à peu et avec quelque habileté arriverons-nous à tout concentrer entre nos mains, mais il faut aller progressivement ", écrivait Albert Thomas au chef du Service des réfugiés un mois plus tôt ${ }^{24}$. À partir de la fin février 1925, le placement des réfugiés russes en France par l'entremise du BIT donne lieu à des réunions mensuelles qui associent les représentants du BIT, des ministères du Travail et de l'Agriculture, du ministère des Affaires 
étrangères, et de la SGI. Lors de ces réunions, le BIT présente des demandes de transfert pour les réfugiés russes et le ministère du Travail émet des avis.

Pour Albert Thomas, ce système est avantageux pour tous les acteurs (États de départ, États d'immigration, employeurs, réfugiés sans emploi). Il écrit: "Ainsi, tout le recrutement se trouve centralisé et contrôlé de façon à empêcher tout monopole au bénéfice d'une organisation particulière de réfugiés d'un caractère politique ou l'exploitation de réfugiés par des intermédiaires ou autrement $\aleph^{25}$. Le souci de contourner les clivages politiques de l'émigration russe et de soustraire les réfugiés à la toute puissance des recruteurs de main-d'œuvre clandestine qui sévissent à Constantinople, Beyrouth ou Belgrade sont liés. Cette préoccupation est partagée par les milieux associatifs d'action sociale d'inspiration religieuse ou laïque ou encore communautaire. Ainsi, Lucien Wolf, fondateur avec Louis Varlez en 1924 de la Conférence Permanente internationale des Organisations privées pour la Protection des Migrants (CPPM), partenaire du BIT, est présent lors des conférences intergouvernementales sur les questions migratoires de 1924 à Rome et de 1928 à La Havane. Certaines de ses propositions prennent la forme de recommandations dans le cadre des arrangements adoptés (question des familles séparées, de l'inspection des émigrants à bord des navires, des cartes pour émigrants en transit...). La question de la protection des réfugiés et apatrides est intégrée à la réflexion menée par la CPPM et fait l'objet d'études particulières dans ces mêmes années.

La collaboration avec la SGI n'est cependant pas sans ambiguïtés. Cette structure, fondée en 1924 par l'unification de diverses associations patronales privées, avec pour mission de recruter des travailleurs étrangers, a des liens étroits avec l'administration, en particulier le ministère du Travail. Ces liens expliquent que Jean Duhamel se présente à Genève dès l'annonce de la mise en place du système de placement. C'est $\mathrm{M}$. Lebelle qui favorise ce rapprochement en 1925. À l'inverse, on sait la réserve, voire l'hostilité de Marcel Paon, à l'égard des procédés de la SGI (Schor, 1985 : 211-220). Le lien étroit existant entre Duhamel et Lebelle se concrétise durant l'été 1926 avec la fondation à Genève de la Société Internationale des Migrations (ci-après SIM). M. Lebelle démissionne alors de ses fonctions au ministère français du Travail pour devenir le directeur général de la SIM. Jean Duhamel en devient le Délégué général et Jacques de Peyerimhoff de Fontenelle (président du comité central des Houillères de France et vice-président du conseil d'administration de la SGI), le Secrétaire général. La SIM a pour objet "en accord avec les autorités compétentes de développer les mouvements migratoires dans le monde par tous les moyens et de s'associer à tous les phénomènes d'émigration et d'immigration $»^{26}$. Cette internationalisation de l'activité de recrutement organisée en France par la SGI est l'objectif de la nouvelle société créée ${ }^{27}$. Il s'agit d'alimenter le marché du travail sud-américain en organisant les recrutements de main-d'œuvre en Europe. Dès le printemps 1926, Jacques de Peyerimhoff et Jacques Chastenet se rendent en Argentine et au Brésil pour une première étude de terrain. Les archives du Service des réfugiés du BIT attestent des intenses négociations qui se déroulent durant l'année 1927 entre la SIM et le BIT au sujet d'un accord global concernant les transferts internationaux de réfugiés. Duhamel et Lebelle proposent de prendre dans chaque convoi d'immigrants à destination de l'Amérique du Sud $35 \%$ de réfugiés. Mais, l'affaire ne se conclura pas. Le problème posé reste comme dans le cas du recrutement en France par la SGI, celui du contrôle des activités de la SIM et des initiatives prises par J. Duhamel. Ainsi, en 1928, Albert Thomas, après un entretien avec Pierre Pouillot, le successeur de M. Lebelle à la tête du 
Service de la main-d'œuvre étrangère au ministère français du Travail, décide de mettre un frein à la collaboration existante avec la SGI et la SIM. Pierre Pouillot se plaint ouvertement des agissements de la SGI et Albert Thomas constate également qu'à plusieurs reprises, J. Duhamel a cherché à « se substituer à nous, à profiter de nos contrats. Il s'est même présenté à plusieurs reprises à $\mathrm{M}$. Pouillot en demandant certaines choses en notre nom $»^{28}$. La correspondance avec le délégué à Berlin atteste également du peu de contrôle réel du BIT sur le recrutement des réfugiés à destination de la France ${ }^{29}$. Le point de vue d'Albert Thomas rejoint en la matière celui de Marcel Paon et ressort d'un positionnement plus général sur la politique française d'immigration. Comme l'explique le directeur du BIT dans la préface du livre de Marcel Paon sur la question, l'immigration spontanée, individuelle, libre constitue un danger (Paon, 1926: 8-15). L'immigration doit être réglementée et dans la mesure du possible collective. Une législation cohérente est nécessaire et la création d'un office national d'immigration s'impose.

\section{Réfugiés, chômeurs : catégorisation de la migration ou du travail ?}

La conception qui sous-tend le programme international de placement du BIT en faveur des réfugiés est à rechercher dans les années d'avant-guerre et dans l'expérience française de la Première Guerre mondiale. Elle illustre le transfert au sein de l'organisation internationale des idées avancées dans les milieux réformateurs de la section française de la Conférence internationale du chômage, de l'Association française pour la lutte contre le chômage et de l'office du travail. C'est au sein de ces milieux que s'élaborent en Europe comme aux États-Unis dans la décennie qui précède la guerre la catégorie du chômeur et la définition progressive du phénomène du chômage (Topalov, 1994 : 59-115). Pour la France, Louis Varlez et Max Lazard tiennent une place de premier plan. On trouve également dans l'Association française pour la lutte contre le chômage Arthur Fontaine et Albert Thomas. Au début des années vingt, ces hommes se retrouvent au BIT où ils travaillent ensemble à élaborer des standards internationaux. Ils souhaitent rationaliser le fonctionnement du marché du travail par la convergence entre les nécessités sociales et économiques et favoriser la généralisation des bureaux de placement. Les bases de cette démarche sont posées dès 1919 lors de la Conférence Internationale du Travail de Washington durant laquelle une convention sur le chômage, stipulant que le fonctionnement des différents systèmes nationaux de placement serait coordonné par le BIT, est adoptée. En 1921, la Commission internationale de l'émigration énonce les principes de bases des accords de recrutement collectif. Lors des deux conférences internationales de l'émigration de Rome (1924) et de la Havane (1928), les conditions du recrutement collectif font l'objet de stipulations particulières (protection de la main-d'œuvre du pays d'immigration, sélection des candidats au départ). La convention sur le chômage, adoptée en 1919 est à la fin des années vingt ratifiée par 23 États et le BIT a mis en place dans l'intervalle un système de renseignements sur les demandes d'emplois et d'immigration. Néanmoins les recommandations ou conventions sont peu ou pas appliquées par les pays signataires. Les efforts de coordination menés par le BIT se heurtent à un contexte défavorable et à la difficulté de transformer en pratiques une volonté réformatrice et des analyses macro-sociales. Ainsi, lors de la Conférence Internationale du Travail de 1926, une nouvelle résolution est adoptée par laquelle il est demandé au BIT d'intensifier ses efforts pour développer l'application des mesures envisagées pour 
lutter contre le chômage. Les trois premiers points avancés sont : la communication par les gouvernements des renseignements statistiques concernant le chômage et les mesures adoptées au niveau national (les fameux «baromètres économiques»); le développement des institutions d'assurance contre le chômage et l'admission des étrangers au bénéfice de ces institutions sous réserve de réciprocité ; le développement des institutions publiques de placement et la coordination internationale des divers systèmes de placement.

30 Le raisonnement dominant est alors de dégager l'influence des grands facteurs de caractère international sur le chômage. Parmi eux, l'analyse du déséquilibre existant entre la quantité de main-d'œuvre disponible et la capacité productrice du pays paraît primordiale, l'idée étant que le problème peut être résolu par un transfert de la dite main-d'œuvre. D'où l'association de Louis Varlez à la mission en Amérique du Sud pour le placement des réfugiés en 1925. Le lien entre lutte contre le chômage et transfert des réfugiés est également établi dans le cas des relations nouées entre la Bulgarie et le BIT au milieu des années vingt.

Les réfugiés chômeurs apparaissent à la fois comme ceux qui entravent la mise en place d'un régime de protection sociale et ceux dont la situation personnelle (nonappartenance à la collectivité nationale) permet le mieux l'expérimentation à l'échelle européenne voire mondiale des transferts de main-d'œuvre. D'ailleurs, les conclusions de l'enquête sont formelles: les réfugiés provoquent une hypertrophie du prolétariat urbain, qui entraine de graves perturbations dans les conditions du travail et l'application de la législation sociale : abaissement général des salaires, non-respect de la journée de huit heures dans l'artisanat et les petites entreprises, risque de faillite de l'assurance-chômage à cause de la concurrence faite par les réfugiés à la main-d'œuvre nationale, effets négatifs sur les assurances sociales en raison de la charge plus grande que font peser les réfugiés (plus souvent malades!). Les solutions résident donc essentiellement dans l'émigration des réfugiés russes et arméniens. Albert Thomas, dans la préface du rapport, insiste d'ailleurs sur la nécessité de sauvegarder « au milieu de toutes les crises, le maintien et le développement de la législation ouvrière ». Par ailleurs, la généralisation de l'assurance-chômage et des bureaux de placement voulue par les réformateurs sociaux implique une définition du chômeur et la création d'une catégorie qui se distingue du pauvre, de l'invalide ou du paresseux. Les effets de cette catégorisation sur l'appréhension par le BIT de la question des réfugiés sont multiples. Il s'agit d'abord de l'idée que les réfugiés participent localement aux déséquilibres des marchés nationaux du travail et qu'une approche macro-sociale rationalisatrice s'impose au traitement de la question. Seul le changement d'échelle permet une résolution globale du problème. Tout comme la prise en charge nationale de la protection sociale a pu apporter des réponses à des déséquilibres régionaux et locaux, la gestion par la BIT au niveau international peut être un mode de résolution des tensions nationales créées par les concentrations de réfugiés. Par ailleurs, le problème doit être au préalable posé de manière scientifique et objective, ce que permet la statistique sociale internationale, d'où l'importance accordée à l'établissement d'une comptabilité des réfugiés chômeurs. Enfin, la question n'est plus posée en termes de morale individuelle ou collective comme ont $\mathrm{pu}$ le faire les organisations de bienfaisance religieuses ou laïques dans la phase d'urgence de l'après-guerre. Ce changement de perspective porte en lui la volonté de démanteler la catégorie du réfugié au profit de celui de l'employable. Il reflète un positionnement critique par 
rapport à l'action philanthropique, notamment par rapport à l'assistance par le travail, largement pratiquée dans les camps de réfugiés et orphelinats dans les années vingt. Cette entrée biaisée dans la catégorie du réfugié implique de séparer ceux qui sont employables de ceux qui ne le sont pas. Cette classification tient, comme nous avons pu l'observer, tant à des facteurs propres (capacité physique, disponibilité immédiate, acceptation de la migration) qu'aux besoins et variations du marché du travail des pays d'immigration. De fait, un partage s'instaure entre travailleurs jeunes et valides employables dans l'industrie ou l'agriculture, objets de la catégorie créée par le BIT et le reste des réfugiés qui se regroupe sur des critères mixtes (étudiants, membres des professions libérales, enfants, vieillards, invalides, etc.) voués à rester à la charge des structures d'assistance diverses. D'ailleurs, Albert Thomas le constate dès le rapport annuel de 1925 : « Il y a un autre problème plus difficile [...] et qui n'est pas strictement une question de placement: c'est la question des invalides, des demi-invalides, des personnes âgées et des enfants en âge de fréquenter l'école. On peut se rendre compte de l'importance de cette question si l'on considère que, dans le Royaume des Serbes, Croates et Slovènes seulement, sur un total de 31000 réfugiés russes il n'y en a pas moins de 11219 , soit $36 \%$, qui appartiennent à cette catégorie $»^{30}$. Le chevauchement catégoriel ainsi établi a donc été à la fois partiel et conjoncturel. L'application expérimentale aux réfugiés d'une catégorisation du chômage en cours de généralisation entre 1925 et 1928 par le BIT a eu des résultats limités et le manque de moyens propres n'a permis qu'un contrôle très relatif des recrutements collectifs comme le montre le cas français.

Cette expérience de quelques années s'inscrit enfin dans la réorganisation du mouvement réformateur au lendemain de la guerre et dans la volonté des quelques hommes en poste au BIT de transformer en action des analyses ou expertises d'un petit milieu qui dans la période précédente s'était consacré à construire un champ scientifique et à acquérir une légitimité institutionnelle. Ainsi, la première assemblée générale de l'Association internationale pour la lutte contre le chômage après la guerre ne se tient qu'en septembre 1923 à Luxembourg, dans un contexte marqué par une grave crise économique et une hausse importante du chômage. Or, le constat dominant est que les efforts de l'association ont été couronnés de succès par les conventions et recommandations, concernant la lutte contre le chômage, adoptées par la Conférence internationale du travail de Washington de 1919 et que son programme d'action est "actuellement réalisé par le Bureau International du Travail »13. Ce constat traduit l'idée, partagée par un certain nombre d'associations membres plus d'une décennie après la fondation, que l'institutionnalisation du champ et sa reconnaissance sont un but en soi et que cet objectif a été atteint. Louis Varlez, président de l'association internationale ne dirige-t-il pas une section du BIT? Arthur Fontaine, président d'honneur de l'association française, Albert Thomas, membre de l'association, ne président-ils pas aux destinées du BIT ? C'est ce à quoi, lors de l'assemblée générale de 1923, Albert Thomas répond par la négative et la critique à peine voilée. Max Lazard, dans le programme de l'association française, élaboré en 1924 pour faire suite à la conférence de Luxembourg, évoque ainsi les propositions qu'il présente et qui sont destinées à répondre aux instances du directeur du BIT, « lequel pressait l'assemblée de Luxembourg de dire son mot au sujet de la terrible crise de chômage sévissant en plusieurs pays depuis 1921. Les spécialistes de la matière avaient-ils un remède à proposer? Si oui, qu'attendaient-ils pour parler? $»^{32}$. Et Max Lazard d'ajouter que cette question est toute naturelle sur les lèvres d'un homme d'action, «mais extrêmement 
embarrassante pour ceux à qui elle était posée, puisque qu'aucune réponse collective ne pouvait être faite sans accord sur les causes profondes de la crise ». On en revient là à la démarche adoptée par les milieux réformateurs dès la création de l'association: rassembler les associations membres sur des thématiques réduites, objets d'un relatif consensus. La grave et complexe question des causes du chômage représentait à ce titre un sujet de discorde faisant entrer en jeu de multiples éléments d'ordre économique et monétaire. Lors du Congrès international de politique sociale qui rassemble à Prague en 1924 les membres de l'Association internationale de lutte contre le chômage et de l'Association pour la protection légale des travailleurs, les discussions sont menées par Albert Thomas qui plaide pour que l'on dépasse l'ère de la philanthropie pour entrer dans celle de la "démocratie industrielle». Le congrès, constatant la nécessité de s'attaquer aux causes du chômage, adopte une motion sur la prévention des crises par des mesures économiques nécessaires et prend position en faveur du développement des mouvements migratoires cadrant avec les besoins du marché du travail. La question $\mathrm{du}$ recrutement et du placement des travailleurs étrangers fait l'objet d'une étude de William Oualid qui fait suite à un rapport qu'il avait présenté lors du congrès de Luxembourg concernant le contrôle de la main-d'œuvre ouvrière en France (1923 : 2-9 ; $1925: 253-266)^{33}$. L'approbation que ses analyses avaient reçue à Luxembourg est confirmée lors du congrès de Berne de 1925. Il apparaît ainsi que le modèle français est soumis aux associations membres comme exemple à suivre. Lors du même congrès de 1925, l'Association internationale de lutte contre le chômage et l'Association pour la protection légale des travailleurs fusionnent pour former l'Association internationale de progrès social. L'ancien chancelier d'Autriche Charles Renner en devient le président et Louis Varlez le vice-président. Une Association française de progrès social (constituée par la fusion au niveau national des deux associations précédentes) est créée à Paris quelques mois plus tard et Albert Thomas en prend la présidence. Lors de son assemblée générale de mars 1927, le directeur du BIT, dans son discours inaugural, reprenant l'historique du mouvement réformateur plaide en faveur d'une approche plus prospective de la réalité sociale. Elle se doit d'être "plus en phase avec les problèmes actuels $»^{34}$. C'est donc, dans ce contexte particulier que se déroule le programme de placement des réfugiés par le BIT et l'on peut observer les liens existants entre ce projet, l'influence du modèle français et la tentative plus générale de repenser le positionnement du mouvement réformateur sur la question du chômage dans laquelle il s'inscrit comme une sorte d'exercice pratique.

\section{Conclusion}

33 Il reste à constater les limites propres à ce type de chevauchement catégoriel qui outre la question du critère d'employabilité, pose le problème à moyen terme des périodes de contraction des marchés du travail nationaux. Dès le début des années trente, avec l'extension de la crise à l'Europe, la catégorie du réfugié redevient inassimilable et le champ est partagé entre un juridisme rigide, une stigmatisation qui se manifeste par la reprise de l'argumentaire des vrais/faux réfugiés et un discours éthique qui centre le débat sur le droit de l'asile (Kévonian, 2003 : 40-49) (Fauchille, 1924 : 324). Il est vrai que la perspective du BIT dans les années vingt restait, au même titre que la bienfaisance, en marge du politique tant du point de vue des agissements des États créateurs de réfugiés (discriminations, expulsions, dénationalisation en masse) que des politiques d'asile des pays d'accueil ou encore de la position des organisations communautaires de 
réfugiés. Après la Seconde Guerre mondiale, le statut créé par la Convention de 1951 définit le réfugié par les persécutions dont il a pu être l'objet dans son pays d'origine. La connexion établie avec les principes des droits de l'homme est centrale dans la définition adoptée dans un contexte de Guerre froide "chaude». L'approche par catégories nationales est abandonnée au profit d'une individualisation qui place la question de l'éligibilité, prérogative des États, comme nouveau lieu d'enjeux. L'association aux droits de l'homme exclut l'appartenance à de multiples catégories (par exemple migrant économique et réfugié). Cette conception de la catégorisation est à la jonction de deux évolutions: l'une sur le temps long qui concerne l'individualisation du droit international et l'autre conjoncturelle qui tient au statut du politique avec la perte d'autonomie du champ du droit international au profit de la logique de Guerre froide. Cette approche correspond au modèle aristotélicien de la définition catégorielle: les catégories sont des entités aux frontières clairement délimitées ; l'appartenance d'une entité particulière à une catégorie répond au système du vrai ou faux; les membres d'une même catégorie ont un statut égal. Ce modèle ne résout donc pas la question de l'appartenance à plusieurs catégories. À partir des années 1970-1980 avec les débuts de la crise économique et l'augmentation des flux migratoires, les politiques d'immigration changent et affectent les politiques de l'asile des pays occidentaux. Il n'est donc pas étonnant de voir les législations nationales s'orienter vers une re-qualification des conditions d'éligibilité en multipliant les " conditions nécessaires et suffisantes » qui permettent d'accéder au statut convoité et par la création de "sous-catégories ». De la même manière, on peut ainsi comprendre la rhétorique du migrant qui essaie de se faire passer pour réfugié. Cependant, cette fonction devenue prééminente de la preuve provoque elle-même la construction de récits de vie stéréotypés qui contribuent à accentuer les tensions existantes. Dès sa fondation en 1949, le Conseil de l'Europe, qui repose sur la trilogie droits de l'homme, prééminence du droit et démocratie, œuvre en faveur d'une meilleure application en Europe occidentale de la Convention de Genève en favorisant un rapprochement des législations nationales des pays membres. Après l'échec en 1977 du Haut-Commissariat des Nations Unies pour les Réfugiés dans l'élaboration d'une convention sur l'asile territorial, le Conseil de l'Europe met sur pieds un comité d'experts chargé des aspects juridiques de l'asile, des réfugiés et apatrides (le CAHAR). Le transfert à l'échelon régional, l'Europe occidentale, permet de mettre en lumière les enjeux de l'intégration européenne face à une situation de crise économique et en conséquence du droit de l'asile, dans un contexte d'augmentation des flux de réfugiés ${ }^{35}$. Les crises pétrolières provoquent une réorientation des travaux du CAHAR dans une phase de changement décisif de la politique d'immigration et de l'asile des pays européens. On peut dater de cette époque l'essor du concept du pays de premier asile qui connaît depuis les années 1990 une faveur grandissante. Ce tournant du début des années 1980 est repris à partir de 1987 dans les travaux menés par la CEE sur une harmonisation des politiques d'immigration dans un contexte de fermeture des pays d'accueil alors même que les flux migratoires augmentaient. L'analyse de cette période permet de constater que la codification juridique et la constitution d'une catégorie sont comme dans les années trente un cadre légal d'exclusion des migrants indésirables. Les enjeux actuels au sein de l'Union européenne concernent la mise en commun des politiques d'asile et d'immigration prévue par le traité d'Amsterdam, précisées par le Conseil européen de Tampere de 1999. Les réfugiés statutaires résidents sur le territoire de l'UE sont mentionnés dès 2000 comme une main-d'œuvre qui doit être intégrée dans le débat sur 
le nombre de migrants économiques nécessaires dans les différents secteurs d'emploi en Europe ${ }^{36}$. Une politique européenne de l'immigration devrait, lit-on, tenir compte de tous les types d'immigration. Si, les discours récents constatent que les politiques existantes « d'immigration zéro » qui ont prévalu au cours des trente dernières années, ne sont donc plus adaptées au recul démographique annoncé et aux pénuries sectorielles de main-d'œuvre, il n'en reste pas moins que la communautarisation progressive s'effectue d'abord par une intégration du point de vue des politiques de contrôle des frontières et de lutte contre l'immigration clandestine.

\section{BIBLIOGRAPHIE}

BIT (1929) Le problème du chômage. Quelques aspects internationaux (1920-1928), Études et Documents, Série $C, \mathrm{n}^{\circ} 13,210 \mathrm{p}$.

BRIBOSIA Emmanuelle, RÉA Andréa, Dir. (2002) Les nouvelles migrations : un enjeu européen, Bruxelles, Complexe, $284 \mathrm{p}$.

DESROSIÈRES Alain (2000) La politique des grands nombres. Histoire de la raison statistique, Paris, La Découverte, rééd., $457 \mathrm{p}$.

DUFOIX Stéphane (2000) Fausses évidences. Statut de réfugié et politisation, Revue Européenne des Migrations internationales, 16 (3), pp. 147-164.

FAUCHILLE (P.) «Le droit d'émigration et le droit d'immigration », Revue Internationale du Travail, IX, n 3, 1924.

GAUTIÉ Jérome (2002) De l'invention du chômage à sa déconstruction, Genèses, 46, pp. 60-76.

GUÉRIN Denis (1996) Albert Thomas au BIT (1920-1932). De l'internationalisme à l'Europe, Genève, Institut européen de l'Université de Genève, 95 p. (bibliographie).

KÉVONIAN Dzovinar (2002) Les réfugiés des années vingt : objets singuliers d'une socio-histoire des relations internationales, Matériaux pour l'histoire de notre temps, 65-66, pp. 84-87.

KÉVONIAN Dzovinar (2003) Question des réfugiés, droits de l'homme : éléments d'une convergence pendant l'entre-deux-guerre, Matériaux pour l'histoire de notre temps, $\mathrm{n}^{\circ} 72$, oct.-déc. 2003.

OUALID W. (1923) Le contrôle de la main-d'œuvre étrangère en France, Informations sociales, VII.

OUALID W. (1925) Le recrutement et le placement des travailleurs étrangers, Les documents du travail, n 103-104, nov.-déc.

NOIRIEL Gérard (1997) Représentation nationale et catégories sociales. L'exemple des réfugiés politiques, Genèses, 26, pp. 25-54.

NOIRIEL Gérard (1991) La tyrannie du national : le droit d'asile en Europe, Paris, Calmann-Lévy, 355 p.

Réfugiés et droit de l'asile : perspectives historiques et enjeux actuels. Journée d'étude organisée par l'URMIS le 29 mars 2002 : Partiellement paru dans : Hommes et migrations (2002), 1240, pp. 81-106. 
PAON M. (1926) L'immigration en France, Paris, Payot.

SCHOR Ralph (1985) L'opinion française et les étrangers en France (1919-1939), Publications de la Sorbonne, Paris.

SKRAN Claudena (1995) Refugees in inter-war Europe. The Emergence of a Regime, Oxford, Clarendon Press, 1995, $324 \mathrm{p}$.

SPIRE Alexis (2003) Les réfugiés, une main-d'œuvre à part ? Conditions de séjours et accès au marché du travail des réfugiés en France de 1945 à 1975, in Michel Guillon, Luc Legoux, Emmanuel Ma Mung Èds, L'asile politique entre deux chaises. Droits de l'homme et gestion des flux migratoires, Paris, L'Harmattan, pp. 209-228.

TÉMIME Émile (1991) Émigration « politique » et émigration « économique », in L'émigration politique en Europe aux XIX et XX siècles, École française de Rome, pp. 57-72.

TOPALOV Christian (1994) Naissance du chômeur (1880-1910), Albin Michel, Paris.

TOPALOV Christian Dir. (1999) Laboratoires du nouveau siècle. La nébuleuse réformatrice et ses réseaux en France (1880-1914), Paris, EHESS, 574 p.

VARLEZ Louis (1929) Le problème des migrations et la Conférence de La Havane de 1928, Revue Internationale du Travail, 19, pp. 1-19.

\section{ANNEXES}

\section{Annexes documentaires}


Document 1 : Questionnaire individuel rempli par les réfugiés candidats au placement

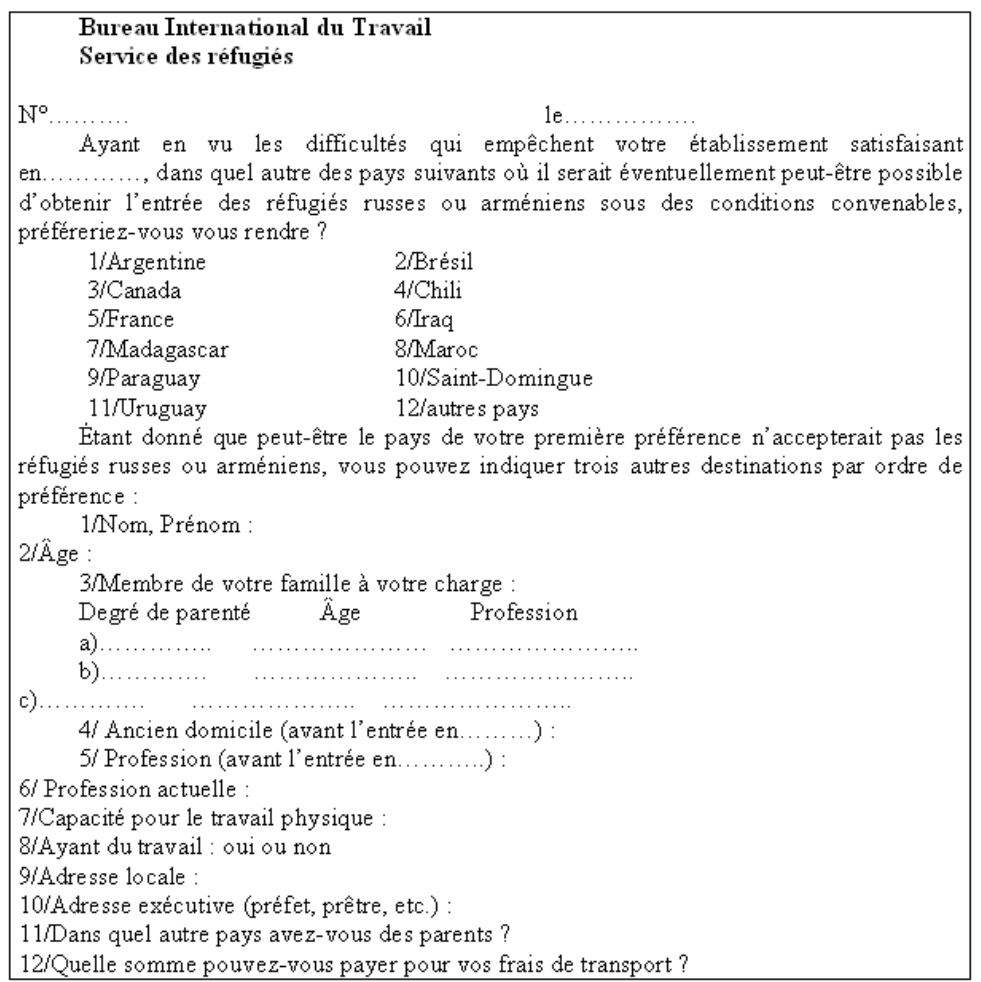

Source : Circulaire-lettre à toutes les délégations du BIT, 30 avril 1926. ASDN, FMN, C1398, R 402/5/0

Tableau 1 : Statistiques du BIT concernant les réfugiés statutaires (russes et arméniens) sans emploi en 1925 et 1927

\begin{tabular}{|c|c|c|c|c|}
\hline \multirow[t]{2}{*}{ Pays } & \multicolumn{2}{|c|}{ Nombre de réfugiés russes et arméniens sans emploi } & \multirow[t]{2}{*}{$\%$} & \multirow[t]{2}{*}{$\%$} \\
\hline & selon le rapport de 1925 & selon le rapport de 1927 & & \\
\hline Allemagne & 83000 & 83000 & 32,3 & 27,8 \\
\hline Autriche & 2509 & 2509 & 1 & $\mathbf{0 , 8}$ \\
\hline Bulgarie & $8800 /$ environ 20000 & 8800 /environ 20000 & 11,2 & 9,6 \\
\hline Chine & 18758 & 18758 & 7,3 & 6,3 \\
\hline Estonie & 2775 & 2775 & 1 & 0,9 \\
\hline Finlande & non mentionnée & 11000 & & 3,7 \\
\hline Grèce & $1640 / 22689$ & $1640 / 23000$ & 9,5 & 8,2 \\
\hline Japon & non mentionnée & 1650 & & 0,5 \\
\hline Lettonie & 1910 & 1910 & 0,7 & 0,6 \\
\hline Pologne & 42655 & 42655 & 16,6 & 14,3 \\
\hline Syrie & environ 25000 & environ 50000 & 9,7 & 16,7 \\
\hline Tchécoslovaquie & non mentionnée & 4000 & & 1,3 \\
\hline Turquie & 2 596/environ 5000 & 2 596/environ 5000 & 3 & 2,5 \\
\hline Yougoslavie & 19301 & 19301 & 7,5 & 6,4 \\
\hline Total & 256633 & 298594 & & \\
\hline
\end{tabular}

Sources : BIT, SDN, rapports annuels de 1925 et 1927. MAE/P, SDN, 1802. A.41.1925; ASDN, FMN, C1470, A.48.1927.VIII. 
Tableau 2 : Bilan des opérations de placements de réfugiés statutaires par le BIT en 1925-1926

\begin{tabular}{|c|c|c|c|}
\hline & $\begin{array}{l}\text { Nombre de réfugiés } \\
\text { russes et arméniens } \\
\text { évacués et placés }\end{array}$ & $\begin{array}{l}\text { Pays principaux } \\
\text { d'origine }\end{array}$ & Pays de destination \\
\hline 1925 & $13053 / 6655$ & \begin{tabular}{|l|} 
Allemagne 4444 \\
Autriche 243 \\
Bulgarie 2100 \\
Chine 700 \\
Estonie 962 \\
Grèce 6655 \\
Lettonie 484 \\
Pologne 1783 \\
Tchecoslovaquie 600 \\
Turquie 946 \\
Yougoslavie 793
\end{tabular} & $\begin{array}{l}\text { destinations diverses dont: } \\
\text { France : } 6693 / 2587 \text {, soit } 47 \% \\
\text { Amérique centrale et du sud: } \\
1231 / 233 \text {, soit } 7,4 \% \\
\text { Arménie soviétique : } 3029\end{array}$ \\
\hline 1926 & $12839 / 2747$ & $\begin{array}{l}\text { Allemagne } 4164 \\
\text { Bulgarie } 3631 \\
\text { Estonie } 888 \\
\text { Grèce } 2119 \\
\text { Lettonie } 593 \\
\text { Pologne } 1073 \\
\text { Yougoslavie } 801\end{array}$ & $\begin{array}{l}\text { destinations diverses dont : } \\
\text { France : } 6292 / 2747 \text {, soit } 58 \% \\
\text { Allemagne : } 2134 \\
\text { (uniquement placés) } \\
\text { Autres pays europeens : } 1229 \text { soit } 8 \% \\
\text { Amérique centrale et du sud : } \\
1792 / 55 \text {, soit } 12 \%\end{array}$ \\
\hline $\begin{array}{l}\text { Total } \\
1925-1926\end{array}$ & 35294 & & dont France : $52 \%$ \\
\hline
\end{tabular}

Source : Résumé statistique du recensement, de l'évacuation et de l'établissement des réfugiés russes et arméniens du 1er janvier 1925 au 15 mai 1927. ASDN, FMN, C1400, R 402/10/0.

Tableau 3 : Bilan des opérations de placements de réfugiés statutaires par le BIT de 1925 à 1928

\begin{tabular}{|c|c|c|c|}
\hline & $\begin{array}{l}\text { Nombre de réfugiés } \\
\text { russes et arméniens } \\
\text { évacués et placés }\end{array}$ & $\begin{array}{l}\text { Pays principaux } \\
\text { d'origine }\end{array}$ & Pays de destination \\
\hline 1927 & environ 3000 & données partielles & données partielles \\
\hline 1928 & environ 11600 & $\begin{array}{l}\text { Allemagne } 659 \\
\text { Autriche } 12 \\
\text { Bulgarie } 552 \\
\text { Chine } 316 \\
\text { Estonie } 647 \\
\text { France } 118 \\
\text { Grèce } 2243 \\
\text { Lettonie } 520 \\
\text { Pologne } 837 \\
\text { Suisse } 2 \\
\text { Tchécoslovaquie } 264 \\
\text { Turquie } 753 \\
\text { Yougoslavie } 655\end{array}$ & $\begin{array}{l}\text { destinations diverses dont: } \\
\text { France, Tunisie, Canada, Amérique du } \\
\text { Sud } \\
\text { (450 princip. en Argentine), etc. : } 7578 \\
\text { Syrie-Liban : env. } 5000 \\
\text { (uniquement placés) }\end{array}$ \\
\hline 1927-1928 & environ 14600 & & \\
\hline $\begin{array}{l}\text { Total } \\
1925 \text { à } 1928\end{array}$ & \multicolumn{3}{|c|}{$\begin{array}{l}\text { environ } 50000 \\
\text { La majorite en France ou par le gouvernement français } \\
\text { (empire colonial, territoires sous mandats) }\end{array}$} \\
\hline
\end{tabular}

Source : BIT, SDN, rapport annuel de 1928. ASDN, FMN, C1471, A.33.1928.VIII. 


\section{NOTES}

1. F. Nansen abandonne en 1924 les tentatives de rapatriement des réfugiés russes en URSS.

2. «L'œuvre de secours aux réfugiés", Revue Internationale du Travail, XII, n 1, janv. 1928, pp. 73-74.

3. Rapport de la Délégation de Berlin, janvier-juillet 1925. Archives de la Société des Nations à Genève (ci-après ASDN), FMN, C1386, R 401/3/2.

4. «L'œuvre de secours aux réfugiés », op. cit., p. 83.

5. Rapport du Dr Heimroth au Major T. F. Johnson, 7 octobre 1926. ASDN, FMN, C1400, R 402/10/5/1.

6. Rapport sur l'activité du Bureau de liaison pour les Affaires des réfugiés russes en Autriche, mai 1926. ASDN, FMN, C1400, R 402/10/5/1.

7. Pour 1926, nouveau constat d'impossibilité de communiquer des chiffres fiables: «Les autorités allemandes ne possèdent pas elles-mêmes, à l'heure actuelle, de renseignements statistiques, comme les représentants du gouvernement allemand vous l'ont communiqué à

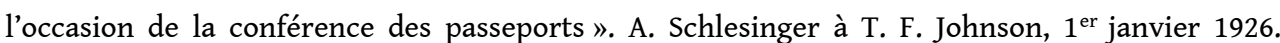
ASDN, FMN, C1398, R 402/5/0.

8. Mémorandum soumis à la conférence intergouvernementale, 10-11 mai 1926. ASDN, FMN, C1470.

9. Réfugié russe : toute personne d'origine russe qui ne jouit pas ou ne jouit plus de la protection du Gouvernement de l'URSS et qui n'a pas acquis une autre nationalité ; Réfugié arménien : toute personne d'origine arménienne auparavant sujette de l'Empire ottoman qui ne jouit pas ou ne jouit plus de la protection du Gouvernement de la République Turque et qui n'a pas acquis une autre nationalité. ASDN, FMN, C1470, doc. A44.1926.R/I.G.C.7-1926.

10. Cette définition est à mettre en rapport avec les pressions des autorités italiennes à la $\mathrm{SdN}$ pour une approche restrictive et la non-prise en considération des émigrés italiens dénationalisés par la loi du 31 janvier 1926.

11. Le paiement de cette taxe prend la forme d'un timbre à l'effigie de F. Nansen apposé annuellement sur le certificat ou le permis de séjour lorsque le pays d'accueil ne dispense pas de certificat.

12. Il n'y aura pas de nouvelles adhésions jusqu'à la Seconde Guerre mondiale.

13. En Allemagne et en Bulgarie, le BIT obtient que les réfugiés russes soient exemptés des mesures de restriction s'appliquant aux travailleurs étrangers et qu'ils bénéficient des indemnités de l'assurance-chômage.

14. En Allemagne, l'une des grandes revendications des organisations de réfugiés était l'extension du certificat à tous les réfugiés arrivés avant le $1^{\mathrm{er}}$ janvier $1923 \mathrm{au}$ lieu du $1^{\mathrm{er}}$ janvier 1922. Cette mesure régularise la situation de très nombreux réfugiés considérés jusque là comme "Staatenlose ", apatrides.

15. Mémorandum du colonel Procter, 9 mars 1925. Archives du ministère français des Affaires étrangères, Paris (ci-après MAE/P), SDN, 1804, ff. 47-52.

16. Pays représentés : Allemagne, Bulgarie, Finlande, France, Grande-Bretagne, Grèce, Lettonie, Pologne, Roumanie, Tchécoslovaquie, Yougoslavie.

17. Résumé statistique du recensement, de l'évacuation et de l'établissement des réfugiés russes et arméniens du $1^{\mathrm{er}}$ janvier 1925 au 15 mai 1927. ASDN, FMN, C1400, R 402/10/0.

18. $36^{\text {ème }}$ session du Conseil d'administration du BIT, mai-juin 1927, p. 51. ASDN, FMN, C1470.

19. Paris, Pédone, p. 57. L'auteur fait ici référence à la situation des réfugiés russes en Allemagne en 1923, dans une période de fort chômage. Les réfugiés ont été les premiers touchés par les politiques de licenciement en particulier dans les grandes villes. Le camp de StralauRummelsberg, situé près de Berlin et destiné aux chômeurs allemands a ainsi connu le 
cantonnement de réfugiés russes sans emplois. Quant aux invalides, enfants et vieillards, ils sont dans cette période recueillis dans les camps de Scheuen et Wünsdorf.

20. SDN, BIT, Rapport d'Albert Thomas pour 1928, p. 11 et appendice IV, p. 25. ASDN, FMN, C 1471, A.33.1928.VIII.

21. Voir les commentaires désabusés d'A. Thomas dans son rapport annuel, ibid., p. 13.

22. R. Schlemmer à Major Johnson, 6 août 1927, confidentiel. ASDN, FMN, C 1402, R 402/10/66/1.

23. Ibid.

24. Note d'Albert Thomas, 20 janvier 1925. ASDN, FMN, C 1410, R 405/1/22/1.

25. Mémoire présenté par le Directeur du BIT, Albert Thomas, 3 sept. 1926. ASDN, FMN, C 1470, A. 44.1926.

26. Statuts de la Société Internationale des migrations. ASDN, FMN, C 1410, R 404/7/59/1, confidentiel.

27. S'y trouvent associés : la Deutsche Bank, la Nord Deutscher Lloyd, la Société de la banque suisse, la Banque italo-belge, Les Chargeurs réunis, La Compagnie générale transatlantique, la Société générale d'Immigration, l'office central de la main d'œuvre agricole, les Houillères de France, etc.

28. Note d'Albert Thomas, 18 juillet 1928. ASDN, FMN, C 1410, R 404/7/59/1.

29. Les contrats sont envoyés directement par J. Duhamel à M. de Bellegarde, en sa qualité de président du Zentralbüro russicher Arbeitgenossenschaften. Le délégué du BIT ne s'occupe lui que de pourvoir les réfugiés des papiers nécessaires. Sur le parcours, les représentants de la SGI encadrent les convois. M. Schlesinger à Major Johnson, 28 octobre 1926. ASDN, FMN, C 1401, R $402 / 10 / 24 / 1$.

30. SDN et BIT, Rapport sur l'œuvre en faveur des réfugiés, sept. 1925, pp. 2-3. MAE/P, SDN, 1802, f. 22.

31. «L'Assemblée générale de l'Association internationale de lutte contre le chômage », Revue Internationale du Travail, VIII, n 5, nov. 1923, pp. 742-748.

32. Les documents du travail, $n^{\circ} 88-89$, août-septembre 1924, p. 8.

33. Voir bibliographie.

34. Les documents du travail, $\mathrm{n}^{\circ}$ 120-121, avril-mai 1927. Allocution d'A. Thomas : pp. 81-87.

35. Archives du Conseil de l'Europe (Strasbourg), AS/PR (26) 12.16 décembre 1974.

36. Commission des Communautés européennes, Communication de la Commission au Conseil et au Parlement européen, Une politique communautaire en matière d'immigration, 22 novembre 2000, $\operatorname{COM}(2000) 757$ final.

\section{RÉSUMÉS}

L'histoire de l'action internationale en faveur des réfugiés européens au $x x^{\mathrm{e}}$ siècle est marquée de manière permanente par des enjeux de catégorisation : oscillations entre approche collective et individualisation d'une part et enjeux de catégorisation verticale entre statut de réfugiés et ensemble plus vaste des migrants de l'autre. Considérant les réfugiés comme objets singuliers d'une socio-histoire des relations internationales, cette étude propose de mettre en lumière une expérience originale menée dans les années vingt en faveur des réfugiés apatrides russes et arméniens, alors seuls groupes juridiquement reconnus par la Société des Nations et dotés d'un statut propre. Ce statut fondé sur une situation légale pénalisante - l'absence de nationalité, et 
rapportée à une causalité globalisante - la guerre mondiale, se conçoit comme l'institutionnalisation d'une marginalité administrative et sociale. Or, l'expérience menée entre 1925 et 1929 consiste à transférer au Bureau International du Travail l'action internationale en faveur de ces réfugiés dans la perspective de leur placement professionnel et de fait à favoriser un chevauchement catégoriel entre réfugié et migrant économique. Comme on peut l'observer aujourd'hui (la remarque est également valable pour les années trente), ce chevauchement catégoriel a accentué en d'autres temps la fragilité structurelle du statut de l'exilé en introduisant l'argumentaire du vrai/faux réfugié. Par ailleurs, il ne présuppose pas une meilleure considération du réfugié qui reste de tout temps stigmatisé et en butte à la suspicion du travestissement catégoriel. En analysant l'approche qui domine au BIT, l'action menée et ses résultats, cette étude veut mettre en lumière la variabilité de l'assignation identitaire en fonction de facteurs externes et les conditions de malléabilité de catégories juridiquement rigides.

Categories and International Migrations: International Labor Organisation and Refugees (1925-1929)

In the course of the history of international action in the 20th century, there has always been the issue of categories, with variations between collective and individual approaches, between refugees and economically motivated migrants. After World War I, the League of Nations conferred upon Russian and Armenian stateless refugees a special judicial status. This status recognized that statelessness in Europe had become more common, and it represented the first in a long series of legal measures designed to protect stateless persons and refugees. From 1925 to 1929 , the League of Nations appointed the International Labor Organisation to help refugees, as they had economic migrants, gain access to employment. What we see today was also true in the 1930's: the merging of the two categories - refugees and economically motivated migrants was risky for refugees and for the recognition of the validity of their claim to international protection (the question of real/false refugee). This study presents the ILO's policy and its outcome: statistical work, a new system of refugee employment, relations with European states, in particular with France.

Categorías y migraciones internacionale : La Organización Internacional del Trabajo y los refugiados (1925-1929)

La historia de la acción internacional del siglo XX a favor de los refugiados europeos ha estado permanentemente marcada por cuestiones de categorización : las oscilaciones entre una visión colectiva et individualista, por un lado, y la categorización vertical entre el estatuto de refugiado y el del conjunto de inmigrantes, por otro. Considerando a los refugiados como objetos singulares de la socio-historia de las relaciones internacionales, este estudio expone una experiencia original realizada durante los años veinte en dirección de refugiados rusos y armenios apátridas. Amparados por el reconocimiento jurídico de la Sociedad de Naciones, estos refugiados eran los únicos en los años veinte que disponían de un estatuto propio. Como consecuencia de la Guerra Mundial, rusos y armenios se hallaban en una situación legal desfavorable (ausencia de nacionalidad). El estatuto propio que se les otorgó fue concebido como la institucionalización de una marginación administrativa y social. Así, la experiencia realizada entre 1925 y 1929 consistió en transferir a la Organización Internacional del Trabajo (OIT) la acción internacional en dirección de estos refugiados de cara a proponerles una salida profesional y favorecer así la fusión de las categorías de refugiado y de inmigrante económico. Actualmente se puede observar (observación también valida para los anos treinta) que esta fusión de categorías ha acentuado la fragilidad estructural del estatuto de exiliado debido a la introducción del concepto de verdadero/falso refugiado. Esta situación no presupone tampoco que el refugiado sea mejor considerado ya que, además de seguir sufriendo la estigmatización, la acusación de « travestismo de categorías » pesa sobre él permanentemente. A partir del análisis de la visión dominante en la OIT, de la experiencia realizada y de los resultados obtenidos, este estudio evidencia la 
variabilidad de la asignación de identidades; variabilidad que está en función de factores externos y del margen de maleabilidad de categorías jurídicamente rígidas.

INDEX

Mots-clés : Arméniens, marginalisation, réfugiés, Russes, statut juridique

\section{AUTEUR}

\section{DZOVINAR KÉVONIAN}

Maître de conférence, Université Paris-X. dzovinar.kevonian@wanadoo.fr 